ESAIM: PROCEEDINGS AND SURVEYS, December 2017, Vol. 58, p. 40-57

Stéphane DELLACHERIE, Gloria FACCANONI, Bérénice GREC, Frédéric LAGOUTIERE, Yohan PENEL

\title{
RELATIVE ENTROPY FOR COMPRESSIBLE NAVIER-STOKES EQUATIONS WITH DENSITY DEPENDENT VISCOSITIES AND VARIOUS APPLICATIONS
}

\author{
Didier Bresch ${ }^{1}$, Pascal Noble ${ }^{2}$ and Jean-Paul Vila ${ }^{3}$
}

\begin{abstract}
This paper provides the full proof of the results announced by the authors in $[C$. R. Acad. Sciences (2016)]. We introduce an original relative entropy for compressible Navier-Stokes equations with density dependent viscosities and discuss some possible applications such as inviscid limit or low Mach number limit. We first consider the case $\mu(\varrho)=\mu \varrho$ and $\lambda(\varrho)=0$ and a pressure law under the form $p(\varrho)=a \varrho^{\gamma}$ with $\gamma>1$, which corresponds in particular to the formulation of the viscous shallow water equations. We present some mathematical results related to the weak-strong uniqueness, the convergence to a dissipative solution of compressible or incompressible Euler equations. Moreover, we show the convergence of the viscous shallow water equations to the inviscid shallow water equations in the vanishing viscosity limit and further prove convergence to the incompressible Euler system in the low Mach limit. This extends results with constant viscosities recently initiated by E. FEIREISL, B.J. Jin and A. Novotny in [J. Math. Fluid Mech. (2012)].
\end{abstract}

\section{INTRODUCTION}

Since the pioneering works of C. DAFERmos [8] and of H.-T. YAU [23], relative entropy methods have become a crucial and widely used tool in the study of asymptotic limits and long-time behavior for nonlinear PDEs.

In a recent paper E. Feireisl, B.J. Jin, A. Novotny (see [10]) have introduced relative entropies, suitable weak solutions and weak-strong uniqueness for the compressible Navier-Stokes equations with constant viscosities. The interested reader is referred to [18], [9] and references cited therein. Based on such relative entropies, various papers have been dedicated to singular perturbations, see the interesting book [11], the articles [1], [20] for instance. See also the recent interesting work by Th. Gallouët, R. Herbin, D. Maltese, A. Novotny in [12] where relative entropy technics are developed to obtain error estimates for a numerical approximation of the compressible Navier-Stokes equation with constant viscosities.

Here we focus on the extension of the results by [10], [1] and [20] to the compressible Navier-Stokes equations with degenerate viscosities depending on the density. This extension is not straightforward: relative entropy for the one-dimensional compressible Navier-Stokes equations with degenerate density dependent viscosity has been, for instance, recently studied by B. НАSPOT in [14] under the rather restrictive assumption that the viscosity function $\mu(\varrho)$ equal to the pressure law $p(\varrho)$ up to a multiplicative constant. The main objective is to get rid of this hypothesis and to extend the result to the multi-dimensional in space case. For that

${ }^{1}$ Laboratoire de Mathématiques UMR5127 CNRS Bâtiment le Chablais Université de Savoie Mont-Blanc 73376 Le Bourget du lac, e-mail: Didier.Bresch@univ-savoie.fr

2 Institut de Mathématiques de Toulouse, UMR5219, Université de Toulouse, CNRS, INSA, F-31077 Toulouse, France, email: Pascal.Noble@math.univ-toulouse.fr

3 Institut de Mathématiques de Toulouse, UMR5219, Université de Toulouse, CNRS, INSA, F-31077 Toulouse, France, email: vila@insa-toulouse.fr

(C) EDP Sciences, SMAI 2017 
purpose, we will take advantage of the $\kappa$-entropy introduced recently by the first author, B. DESJARDINS and E. ZATORSKA in [5]. We introduce a new relative entropy based on a monotonicity property which allows to relax the relation between the viscosity and the pressure asked in [14]. In particular, we are able to handle the case $\mu(\varrho)=\mu \varrho$ and a general pressure $p(\varrho)$ strictly monotone like $p(\varrho)=a \varrho^{\gamma}$ with $\gamma>1$. This corresponds to the compressible Navier Stokes system considered by A. VAsseur and C. YU in [22]. By a combination of estimates based on the so called B-D (Bresch-Desjardin) entropy [4], Mellet-Vasseur estimates [17] and some original renormalization techniques, they recently obtained the first existence result of global weak solutions without additional regularizing/damping terms (friction, surface tension). The readers are also referred to an other approach by J. LI and Z. XIN in [15]. Note that such relative entropy will be used to design appropriate schemes for the compressible Navier-Stokes equation with degenerate viscosities in [6] and prove convergence.

As an application of our relative entropy estimate, we present some mathematical results related to the weakstrong uniqueness, the convergence to a dissipative solution of compressible or incompressible Euler equations. In particular, this mathematically justify the convergence of the solutions of the viscous shallow-water system to the solutions of inviscid shallow-water system or to the incompressible Euler system in the vanishing viscosity and/or low Mach limit. In contrast to constant viscosities, we prove an exponential rate of decay for density dependent viscosities. Finally, we discuss more general viscosities $\mu(\varrho)$ and $\lambda(\varrho)$ assuming an algebraic relation on the coefficients that was introduced by the first author and B. DESJARDINs in [3].

For the reader convenience, let us recall the compressible Navier-Stokes equation with density dependent viscosities:

$$
\left\{\begin{array}{l}
\partial_{t} \varrho+\operatorname{div}(\varrho \mathbf{u})=0 \\
\partial_{t}(\varrho \mathbf{u})+\operatorname{div}(\varrho \mathbf{u} \otimes \mathbf{u})+\nabla p(\rho)-\operatorname{div}(2 \mu(\varrho) D(\mathbf{u}))-\nabla(\lambda(\varrho) \operatorname{div} \mathbf{u})=0
\end{array}\right.
$$

In this paper, we assume an additional algebraic relation introduced by the first author and B. Desjardins in [3]: $\lambda(\varrho)=2\left(\mu^{\prime}(\varrho) \varrho-\mu(\varrho)\right)$.

It has been observed in [5] that this system may be reformulated through an augmented system. Introducing the intermediate velocity $\mathbf{u}+2 \kappa \nabla \varphi(\varrho)$ with $\varphi^{\prime}(s)=\mu^{\prime}(s) / s$, a drift velocity $2 \sqrt{\kappa(1-\kappa)} \nabla \varphi(\varrho)$ and a mixture coefficient $\kappa$, the augmented versions are:

i) Case $\mu(\rho)=\mu \rho, \lambda(\rho)=0$. The augmented system reads

$$
\left\{\begin{aligned}
& \partial_{t} \varrho+\operatorname{div}(\varrho(\mathbf{v}-2 \kappa \mu \nabla \log \varrho))=0 \\
& \partial_{t}(\varrho \mathbf{v})+\operatorname{div}(\varrho \mathbf{v} \otimes(\mathbf{v}-2 \kappa \mu \nabla \log \varrho))+\nabla p(\rho)=\mu \operatorname{div}(2 \varrho(1-\kappa) D(\mathbf{v}))+\mu \operatorname{div}(2 \kappa \varrho A(\mathbf{v})) \\
&-\mu \operatorname{div}(2 \sqrt{\kappa(1-\kappa)} \varrho \nabla \mathbf{w}) \\
& \partial_{t}(\varrho \mathbf{w})+\operatorname{div}(\varrho \mathbf{w} \otimes(\mathbf{v}-2 \kappa \nabla \log \varrho))=\mu \operatorname{div}(2 \kappa \varrho \nabla \mathbf{w})-\mu \operatorname{div}\left(2 \sqrt{\kappa(1-\kappa)} \varrho \nabla \mathbf{v}^{T}\right)
\end{aligned}\right.
$$

with $\mathbf{v}=\mathbf{u}+2 \kappa \mu \nabla \log \varrho$ and $\mathbf{w}=2 \sqrt{\kappa(1-\kappa)} \mu \nabla \log \varrho$. The associated energy estimate (named $\kappa$-entropy) reads for all $t \in[0, T]$ :

$$
\begin{aligned}
& \sup _{\tau \in[0, t]}\left[\int_{\Omega} \varrho\left(\frac{|\mathbf{v}|^{2}}{2}+\frac{|\mathbf{w}|^{2}}{2}\right)(\tau) \mathrm{d} x+\int_{\Omega} F(\varrho)(\tau) \mathrm{d} x\right] \\
& +2 \mu \int_{0}^{t} \int_{\Omega} \varrho\left(\kappa|A(\mathbf{v})|^{2}+|D(\sqrt{1-\kappa} \mathbf{v})-\nabla(\sqrt{\kappa} \mathbf{w})|^{2}\right) \\
& \quad+2 \kappa \mu \int_{0}^{t} \int_{\Omega} \frac{p^{\prime}(\varrho)}{\varrho}|\nabla \varrho|^{2} d x \mathrm{~d} x \leq \int_{\Omega} \varrho\left(\frac{|\mathbf{v}|^{2}}{2}+\frac{|\mathbf{w}|^{2}}{2}\right)(0) \mathrm{d} x+\int_{\Omega} F(\varrho)(0) \mathrm{d} x
\end{aligned}
$$


ii) Case $\mu(\rho)$ and $\lambda(\rho)=2\left(\mu^{\prime}(\varrho) \varrho-\mu(\varrho)\right)$. By introducing $\varphi$ such that $\varphi^{\prime}(s)=\mu^{\prime}(s) / s$, one finds

$$
\left\{\begin{array}{r}
\partial_{t} \varrho+\operatorname{div}(\varrho(\mathbf{v}-2 \kappa \nabla \varphi))=0, \\
\partial_{t}(\varrho \mathbf{v})+\operatorname{div}(\varrho \mathbf{v} \otimes(\mathbf{v}-2 \kappa \nabla \varphi))+\nabla p(\rho) \\
=\operatorname{div}(2 \mu(\rho)(1-\kappa) D(\mathbf{v}))+\operatorname{div}(2 \kappa \mu(\rho) A(\mathbf{v}))-\operatorname{div}(2 \sqrt{\kappa(1-\kappa)} \mu(\rho) \nabla \mathbf{w}) \\
\quad+\nabla\left(\left(\lambda(\varrho)-2 \kappa\left(\mu^{\prime}(\varrho) \varrho-\mu(\varrho)\right) \operatorname{divu}\right),\right. \\
\begin{array}{r}
\partial_{t}(\varrho \mathbf{w})+\operatorname{div}(\varrho \mathbf{w} \otimes(\mathbf{v}-2 \kappa \nabla \varphi(\rho))) \\
=\operatorname{div}(2 \kappa \mu(\rho) \nabla \mathbf{w})-\operatorname{div}\left(2 \sqrt{\kappa(1-\kappa)} \mu(\rho) \nabla \mathbf{v}^{T}\right)-\nabla\left(\left(\mu^{\prime}(\varrho) \varrho-\mu(\varrho)\right) \operatorname{divu}\right)
\end{array}
\end{array}\right.
$$

with $\mathbf{v}=\mathbf{u}+2 \kappa \nabla \varphi(\varrho)$ and $\mathbf{w}=2 \sqrt{\kappa(1-\kappa)} \nabla \varphi(\varrho)$. The associated energy estimate (named $\kappa$-entropy) reads for all $t \in[0, T]$

$$
\begin{gathered}
\sup _{\tau \in[0, t]}\left[\int_{\Omega} \varrho\left(\frac{|\mathbf{v}|^{2}}{2}+\frac{|\mathbf{w}|^{2}}{2}\right)(\tau) \mathrm{d} x+\int_{\Omega} F(\varrho)(\tau) \mathrm{d} x\right] \\
+2 \kappa \int_{0}^{t} \int_{\Omega} \mu(\varrho)|A(\mathbf{v})|^{2} \mathrm{~d} x \mathrm{~d} s+2 \kappa \int_{0}^{t} \int_{\Omega} \frac{\mu^{\prime}(\varrho) p^{\prime}(\varrho)}{\varrho}|\nabla \varrho|^{2} \mathrm{~d} x \mathrm{~d} s \\
+2 \int_{0}^{t}\left[\int_{\Omega} \mu(\varrho)|D(\sqrt{1-\kappa} \mathbf{v})-\nabla(\sqrt{\kappa} \mathbf{w})|^{2} \mathrm{~d} x\right. \\
\left.\left.+\int_{\Omega}\left(\mu^{\prime}(\varrho) \varrho-\mu(\varrho)\right) \mid \operatorname{div}(\sqrt{1-\kappa} \mathbf{v})-\nabla(\sqrt{\kappa} \mathbf{w})\right)\left.\right|^{2} \mathrm{~d} x\right] \mathrm{d} s \\
\leq \int_{\Omega} \varrho\left(\frac{|\mathbf{v}|^{2}}{2}+\frac{|\mathbf{w}|^{2}}{2}\right)(0) \mathrm{d} x+\int_{\Omega} F(\varrho)(0) \mathrm{d} x
\end{gathered}
$$

The paper will be divided in six sections. In the first section, we establish the relative entropy for system 2 , then we prove a weak-strong uniqueness result. The third section corresponds to the convergence of the global $\kappa$ weak solution sequences of the viscous compressible Navier-Stokes system to dissipative solution of the compressible Euler system. The fourth section concerns the convergence to the incompressible Euler equations. In the last section we discuss possible generalization to more general viscosities satisfying the algebraic relation introduced by the first author and B. Desjardins.

\section{Definition of $\kappa$-Entropy SOlution.}

In what follows, we set $\Omega \subset \mathbb{R}^{d}$ an open subset or $\Omega=\mathbb{T}^{d}$ a periodic box. Let us recall here the definition of $\kappa$-entropy solution for the compressible Navier-Stokes equation with degenerate viscosities as introduced recently in [5]

Definition 1. Let $T>0$ and $\kappa$ be such that $0<\kappa<1$, the couple of functions $(\varrho, \mathbf{u})$ is called a global $\kappa-$ entropy solution to compressible Navier-Stokes system with degenerate viscosities if the following properties are satisfied:

- The mass equation is satisfied in the following sense

$$
-\int_{0}^{T} \int_{\Omega} \varrho \partial_{t} \xi \mathrm{d} x d \tau-\int_{0}^{T} \int_{\Omega} \varrho \mathbf{u} \cdot \nabla \xi \mathrm{d} x d \tau=\int_{\Omega} \varrho^{0} \xi(0) \mathrm{d} x
$$

for all $\xi \in C_{c}^{\infty}([0, T) \times \Omega)$. 
- The momentum equation is satisfied in the following sense

$$
\begin{aligned}
& -\int_{0}^{T} \int_{\Omega} \varrho \mathbf{u} \cdot \partial_{t} \boldsymbol{\phi} \mathrm{d} x d \tau-\int_{0}^{T} \int_{\Omega}(\varrho \mathbf{u} \otimes \mathbf{u}): \nabla \boldsymbol{\phi} \mathrm{d} x d \tau+\int_{0}^{T} \int_{\Omega} 2 \mu(\varrho) D(\mathbf{u}): \nabla \boldsymbol{\phi} \mathrm{d} x d \tau \\
& \quad+\int_{0}^{T} \int_{\Omega} \lambda(\varrho) \operatorname{div} \mathbf{u} \operatorname{div} \boldsymbol{\phi} \mathrm{d} x d \tau-\int_{0}^{T} \int_{\Omega} p(\varrho) \operatorname{div} \boldsymbol{\phi} \mathrm{d} x d \tau=\int_{\Omega} \varrho^{0} \mathbf{u}^{0} \cdot \boldsymbol{\phi}(0) \mathrm{d} x
\end{aligned}
$$

for all $\boldsymbol{\phi} \in\left(C_{c}^{\infty}([0, T) \times \Omega)\right)^{3}$.

- Moreover $(\varrho, \mathbf{u})$ satisfies, for all $t \in[0, T]$, the following $\kappa$-entropy estimates

$$
\begin{gathered}
\sup _{t \in[0, T]}\left[\int_{\Omega} \varrho\left(\frac{|\mathbf{u}+2 \kappa \nabla \varphi(\varrho)|^{2}}{2}+\frac{|2 \sqrt{(1-\kappa) \kappa} \nabla \varphi(\varrho)|^{2}}{2}\right)(t) \mathrm{d} x+\int_{\Omega} \varrho e(\varrho)(t) \mathrm{d} x\right] \\
+2 \kappa \int_{0}^{T} \int_{\Omega} \mu(\varrho)|A(\mathbf{u})|^{2} \mathrm{~d} x \mathrm{~d} s+2 \kappa \int_{0}^{T} \int_{\Omega} \frac{\mu^{\prime}(\varrho) p^{\prime}(\varrho)}{\varrho}|\nabla \varrho|^{2} \mathrm{~d} x \mathrm{~d} s \\
+2(1-\kappa) \int_{0}^{T}\left[\int_{\Omega} \mu(\varrho)|D(\mathbf{u})|^{2} \mathrm{~d} x+\int_{\Omega}\left(\mu^{\prime}(\varrho) \varrho-\mu(\varrho)\right)|\mathrm{div} \mathbf{u}|^{2} \mathrm{~d} x\right] \mathrm{d} s \\
\leq \int_{\Omega} \varrho\left(\frac{|\mathbf{u}+2 \kappa \nabla \varphi(\varrho)|^{2}}{2}+\frac{|2 \sqrt{(1-\kappa) \kappa} \nabla \varphi(\varrho)|^{2}}{2}\right)(0) \mathrm{d} x+\int_{\Omega} \varrho_{0} e\left(\varrho_{0}\right) \mathrm{d} x
\end{gathered}
$$

with $\varphi^{\prime}(s)=\mu^{\prime}(s) / s, A(\mathbf{u})=\frac{1}{2}\left(\nabla \mathbf{u}-\nabla^{t} \mathbf{u}\right)$ and the internal energy $e(\varrho)$ defined by

$$
\frac{\varrho^{2} \mathrm{~d} e(\varrho)}{\mathrm{d} \varrho}=p(\varrho)
$$

\section{Relative entropy.}

In this section, we assume $\mu(\rho)=\mu \rho$ and we derive a relative entropy between a weak solution $(\rho, v, w)$ of the augmented system $(2)$ and any other state $(r, V, W)$ of the fluid. Let us consider the relative energy functional, denoted $E(\rho, \mathbf{v}, \mathbf{w} \mid r, V, W)$, defined by

$$
E(\rho, \mathbf{v}, \mathbf{w} \mid r, V, W)=\frac{1}{2} \int_{\Omega} \varrho\left(|\mathbf{w}-W|^{2}+|\mathbf{v}-V|^{2}\right)+\int_{\Omega}\left(F(\varrho)-F(r)-F^{\prime}(r)(\varrho-r)\right)
$$

which measures the distance between a $\kappa$-entropic weak solution $(\varrho, \mathbf{v}, \mathbf{w})$ to any smooth enough test function $(r, V, W)$. We can prove that any weak solution $(\rho, \mathbf{v}, \mathbf{w})$ of the augmented system satisfies the following so-called 
relative entropy inequality

$$
\begin{aligned}
& E(\rho, v, w \mid r, V, W)(\tau)-E(\rho, v, w \mid r, V, W)(0) \\
& +2 \kappa \mu \int_{0}^{\tau} \int_{\Omega} \varrho|A(\mathbf{v}-V)|^{2}+2 \mu \int_{0}^{\tau} \int_{\Omega} \varrho|D(\sqrt{(1-\kappa)}(\mathbf{v}-V)-\sqrt{\kappa}(\mathbf{w}-W))|^{2} \\
& +2 \kappa \mu \int_{0}^{\tau} \int_{\Omega} \varrho\left[p^{\prime}(\varrho) \nabla \log \varrho-p^{\prime}(r) \nabla \log r\right] \cdot[\nabla \log \varrho-\nabla \log r] \\
& \leq \int_{0}^{\tau} \int_{\Omega} \varrho\left(\left(\left(\mathbf{v}-\sqrt{\frac{\kappa}{(1-\kappa)}} \mathbf{w}\right) \cdot \nabla W\right) \cdot(W-\mathbf{w})+\left(\left(\mathbf{v}-\sqrt{\frac{\kappa}{(1-\kappa)}} \mathbf{w}\right) \cdot \nabla V\right) \cdot(V-\mathbf{v})\right) \\
& +\int_{0}^{\tau} \int_{\Omega} \varrho\left(\partial_{t} W \cdot(W-\mathbf{w})+\partial_{t} V \cdot(V-\mathbf{v})\right) \\
& +\int_{0}^{\tau} \int_{\Omega}^{\tau} \partial_{t} F^{\prime}(r)(r-\varrho)-\int_{0}^{\tau} \int_{\Omega} \nabla F^{\prime}(r) \cdot\left[\varrho\left(\mathbf{v}-\sqrt{\frac{\kappa}{(1-\kappa)}} \mathbf{w}\right)-r\left(V-\sqrt{\frac{\kappa}{(1-\kappa)}} W\right)\right] \\
& +\int_{0}^{\tau} \int_{\Omega}^{\tau}(p(r)-p(\varrho)) \operatorname{div}\left(V-\sqrt{\frac{\kappa}{(1-\kappa)}} W\right) \\
& -\kappa \int_{0}^{\tau} \int_{\Omega} p^{\prime}(\varrho) \nabla \varrho \cdot\left[2 \mu \frac{\nabla r}{r}-\frac{1}{\sqrt{(1-\kappa) \kappa}} W\right] \\
& \left.+2 \mu \int_{0}^{\tau} \int_{\Omega} \varrho(D(\sqrt{(1-\kappa)} V)-\nabla(\sqrt{\kappa} W))\right):(D(\sqrt{(1-\kappa)}(V-\mathbf{v}))-\nabla(\sqrt{\kappa}(W-\mathbf{w}))) \\
& +2 \kappa \mu \int_{0}^{\tau} \int_{\Omega}^{\varrho A(V): A(V-\mathbf{v})+2 \kappa \mu \int_{0}^{\tau}} \int_{\Omega} \frac{\sigma}{r} p^{\prime}(r) \nabla r \cdot\left(\frac{\nabla r}{r}-\frac{\nabla \varrho}{\varrho}\right) \\
& +2 \sqrt{\kappa(1-\kappa) \mu} \int_{0}^{\tau} \int_{\Omega} \varrho[A(W): A(\mathbf{v}-V)-A(\mathbf{w}-W): A(V)]
\end{aligned}
$$

for all $\tau \in[0, T]$ and for any pair of test functions

$$
r \in \mathcal{C}^{1}([0, T] \times \bar{\Omega}), \quad r>0, \quad V, W \in \mathcal{C}^{1}([0, T] \times \bar{\Omega}) .
$$

Note that the right hand-side is well defined using the global weak regularity of $(\varrho, \mathbf{v}, \mathbf{w})$. This is the analogue for the density dependent Navier-Stokes equations of what has been proven for the constant viscosity barotropic Navier-Stokes equations in [10]. Note also the presence of the additional new term

$$
\int_{0}^{\tau} \int_{\Omega} \varrho\left[p^{\prime}(\varrho) \nabla \log \varrho-p^{\prime}(r) \nabla \log r\right] \cdot[\nabla \log \varrho-\nabla \log r]
$$

which has a priori no sign but that we will rewrite later in an appropriate manner. This is indeed the corner-stone of our study. The introduction of this term allows to relax the restrictive assumptions between the viscosity $\mu$ and the pressure law $p$ made in [14]. More precisely, we prove the following result:

Theorem 1. Let $\Omega$ be a periodic box. Suppose that the pressure law satisfies $p(\varrho)=a \varrho^{\gamma}$ with $\gamma>1$. Let $(\varrho, \mathbf{u})$ be a finite $\kappa$-entropy solution to the compressible Navier-Stokes system with degenerate viscosity $\mu(\varrho)=\mu \varrho$ and $\lambda(\varrho)=0$ in the sense of definition 1 . Then $(\varrho, \mathbf{u})$ satisfy the relative entropy inequality (9) for any $r \in \mathcal{C}^{1}([0, T] \times \bar{\Omega})$ with $r>0$ and $V, W \in \mathcal{C}^{1}([0, T] \times \bar{\Omega})$.

We explain after the proof how to relax by a density argument the required regularity for the test functions. Remark that existence of $\kappa$-entropic solution for the compressible Navier-Stokes equations with $\mu(\varrho)=\mu \varrho$ with $\mu$ constant and $\lambda(\varrho)=0$ without extra terms (capillary, drag, singular pressure) has been recently proved in the nice paper [22]. 
Proof of the relative entropy estimate. Let us write

$$
\begin{aligned}
& E(\varrho, \mathbf{v}, \mathbf{w} \mid r, V, W)(\tau)-E(\varrho, \mathbf{v}, \mathbf{w} \mid r, V, W)(0) \\
& \left.\left.=\left[\left[\frac{1}{2} \int_{\Omega} \varrho\left(|\mathbf{w}|^{2}+|\mathbf{v}|^{2}\right)\right)+\int_{\Omega} F(\varrho)\right](\tau)-\left[\frac{1}{2} \int_{\Omega} \varrho\left(|\mathbf{w}|^{2}+|\mathbf{v}|^{2}\right)\right)+\int_{\Omega} F(\varrho)\right](0)\right] \\
& +\left[\frac{1}{2} \int_{\Omega} \varrho\left(|V|^{2}+|W|^{2}\right)-\int_{\Omega}\left(F(r)+F^{\prime}(r)(\varrho-r)\right)-\int_{\Omega} \varrho(\mathbf{w} \cdot W+\mathbf{v} \cdot V)\right](\tau) \\
& -\left[\frac{1}{2} \int_{\Omega} \varrho\left(|V|^{2}+|W|^{2}\right)-\int_{\Omega}\left(F(r)+F^{\prime}(r)(\varrho-r)\right)-\int_{\Omega} \varrho(\mathbf{w} \cdot W+\mathbf{v} \cdot V)\right](0)
\end{aligned}
$$

We also calculate that

$$
\begin{aligned}
& 2 \kappa \mu \int_{0}^{\tau} \int_{\Omega} \varrho|A(\mathbf{v}-V)|^{2}+2 \mu \int_{0}^{\tau} \int_{\Omega} \varrho|D(\sqrt{(1-\kappa)}(\mathbf{v}-V)-\sqrt{\kappa}(\mathbf{w}-W))|^{2} \\
& +2 \kappa \mu \int_{0}^{\tau} \int_{\Omega} \varrho\left[p^{\prime}(\varrho) \nabla \log \varrho-p^{\prime}(r) \nabla \log r\right] \cdot[\nabla \log \varrho-\nabla \log r] \\
& =\left[2 \kappa \mu \int_{0}^{\tau} \int_{\Omega} \varrho|A(\mathbf{v})|^{2}+2 \mu \int_{0}^{\tau} \int_{\Omega} \varrho|D(\sqrt{(1-\kappa)} \mathbf{v})-\nabla(\sqrt{\kappa} \mathbf{w})|^{2}+2 \kappa \int_{0}^{\tau} \int_{\Omega} \frac{p^{\prime}(\varrho)}{\varrho}|\nabla \varrho|^{2}\right] \\
& {\left[+2 \kappa \mu \int_{0}^{\tau} \int_{\Omega} \varrho A(V): A(V-\mathbf{v})\right.} \\
& +2 \mu \int_{0}^{\tau} \int_{\Omega} \varrho(D(\sqrt{(1-\kappa)} V)-\nabla(\sqrt{\kappa} W)):(D(\sqrt{(1-\kappa)}(V-\mathbf{v}))-\nabla(\sqrt{\kappa}(W-\mathbf{w}))) \\
& \left.+2 \kappa \mu \int_{0}^{\tau} \int_{\Omega} \frac{\varrho}{r} p^{\prime}(r) \nabla r \cdot\left(\frac{\nabla r}{r}-\frac{\nabla \varrho}{\varrho}\right)\right] \\
& {\left[-2 \kappa \mu \int_{0}^{\tau} \int_{\Omega} \varrho A(\mathbf{v}): A(V)-2 \mu \int_{0}^{\tau} \int_{\Omega} \varrho(D(\sqrt{(1-\kappa)} \mathbf{v})-\nabla(\sqrt{\kappa} \mathbf{w})):(D(\sqrt{(1-\kappa)} V)-\nabla(\sqrt{\kappa} W))\right.} \\
& \left.-2 \kappa \mu \int_{0}^{\tau} \int_{\Omega} p^{\prime}(\varrho) \nabla \varrho \cdot \frac{\nabla r}{r}\right] .
\end{aligned}
$$

Recall the energy estimate satisfied by $(\varrho, \mathbf{v}, \mathbf{w})$

$$
\begin{aligned}
& \left.\left.\left[\left[\frac{1}{2} \int_{\Omega} \varrho\left(|\mathbf{w}|^{2}+|\mathbf{v}|^{2}\right)\right)+\int_{\Omega} F(\varrho)\right](\tau)-\left[\frac{1}{2} \int_{\Omega} \varrho\left(|\mathbf{w}|^{2}+|\mathbf{v}|^{2}\right)\right)+\int_{\Omega} F(\varrho)\right](0)\right] \\
& +\left[2 \kappa \mu \int_{0}^{\tau} \int_{\Omega} \varrho\left[\left.A(\mathbf{v})\right|^{2}+2 \mu \int_{0}^{\tau} \int_{\Omega} \varrho|D(\sqrt{(1-\kappa)} \mathbf{v})-\nabla(\sqrt{\kappa} \mathbf{w})|^{2}+2 \kappa \int_{0}^{\tau} \int_{\Omega} \frac{p^{\prime}(\varrho)}{\varrho}|\nabla \varrho|^{2}\right] \leq 0\right.
\end{aligned}
$$

Let us now test the equations satisfied by $\mathbf{v}$ and $\mathbf{w}$ respectively by $V$ and $W$. This computation is mathematically justified due to the regularity assumptions on $V$ and $W$. Note that we can write the weak formulation satisfied by $\mathbf{w}=2 \sqrt{\kappa(1-\kappa)} \nabla \log \rho$ playing with the regularity satisfied by the $\kappa$ entropy solutions and with 
the weak formulation for the mass equation. We get the following relation

$$
\begin{aligned}
& \int_{\Omega} \varrho(\mathbf{v} \cdot V+\mathbf{w} \cdot W)(\tau)-\int_{\Omega} \varrho(\mathbf{v} \cdot V+\mathbf{w} \cdot W)(0)-\int_{0}^{\tau} \int_{\Omega} \varrho \mathbf{v} \cdot \partial_{t} V-\int_{0}^{\tau} \int_{\Omega} \varrho \mathbf{w} \cdot \partial_{t} W \\
& -\int_{0}^{\tau} \int_{\Omega} \varrho\left[\left(\left(\mathbf{v}-\sqrt{\frac{\kappa}{(1-\kappa)}} \mathbf{w}\right) \cdot \nabla V\right) \cdot \mathbf{v}+\left(\left(\mathbf{v}-\sqrt{\frac{\kappa}{(1-\kappa)}} \mathbf{w}\right) \cdot \nabla W\right) \cdot \mathbf{w}\right] \\
& +2 \kappa \mu \int_{0}^{\tau} \int_{\Omega} \varrho A(\mathbf{v}): A(V)+2 \mu \int_{0}^{\tau} \int_{\Omega} \varrho(D(\sqrt{(1-\kappa)} \mathbf{v})-\nabla(\sqrt{\kappa} \mathbf{w})):(D(\sqrt{(1-\kappa)} V)-\nabla(\sqrt{\kappa} W)) \\
& -\int_{0}^{\tau} \int_{\Omega} p(\varrho) \operatorname{div}\left(V-\sqrt{\frac{\kappa}{1-\kappa}} W\right)+\sqrt{\frac{\kappa}{1-\kappa}} \int_{0}^{\tau} \int_{\Omega} p^{\prime}(\varrho) \nabla \varrho \cdot W \\
& +\int_{0}^{\tau} \int_{\Omega} 2 \sqrt{(1-\kappa) \kappa} \mu \varrho[A(\mathbf{v}): A(W)-A(\mathbf{w}): A(V)]=0
\end{aligned}
$$

Let us now test the mass equation by $|V|^{2} / 2$ and by $|W|^{2} / 2$ and add the two, we get the identity

$$
\begin{aligned}
& \frac{1}{2} \int_{\Omega} \varrho\left(|V|^{2}+|W|^{2}\right)(\tau)-\frac{1}{2} \int_{\Omega} \varrho\left(|V|^{2}+|W|^{2}\right)(0) \\
& =\int_{0}^{\tau} \varrho\left(V \cdot \partial_{t} V+W \cdot \partial_{t} W\right)+\int_{0}^{\tau} \int_{\Omega} \varrho\left(\left(\left(\mathbf{v}-\sqrt{\frac{\kappa}{(1-\kappa)}} \mathbf{w}\right) \cdot \nabla V\right) \cdot V+\left(\left(\mathbf{v}-\sqrt{\frac{\kappa}{(1-\kappa)}} \mathbf{w}\right) \cdot \nabla W\right) \cdot W\right)
\end{aligned}
$$

Remark now that $\partial_{t}\left[F(r)-F^{\prime}(r) r\right]=-\partial_{t} F^{\prime}(r) r$ and thus

$$
-\int_{\Omega}\left[F(r)-F^{\prime}(r) r\right](\tau)+\int_{\Omega}\left[F(r)-F^{\prime}(r) r\right](0)=\int_{\Omega} r \partial_{t} F^{\prime}(r)
$$

Let us now multiply the mass equation by $F^{\prime}(r)$ and integrate in space and time

$$
-\int_{\Omega}\left(F^{\prime}(r) \varrho\right)(\tau)+\int_{\Omega}\left(F^{\prime}(r) \varrho\right)(0)=-\int_{0}^{\tau} \int_{\Omega} \varrho \partial_{t} F^{\prime}(r)-\int_{0}^{\tau} \int_{\Omega} \varrho\left(\mathbf{v}-\sqrt{\frac{\kappa}{(1-\kappa)}} \mathbf{w}\right) \cdot \nabla F^{\prime}(r)
$$

Recalling that $F^{\prime}(r) r-F(r)=p(r)$, we observe that

$$
\begin{aligned}
0 & =\int_{\Omega} \operatorname{div}\left(\left(F(r)-F^{\prime}(r) r\right)\left(V-\sqrt{\frac{\kappa}{(1-\kappa)}} W\right)\right) \\
& =\int_{\Omega} p(r) \operatorname{div}\left(V-\sqrt{\frac{\kappa}{(1-\kappa)}} W\right)+r\left(V-\sqrt{\frac{\kappa}{(1-\kappa)}} W\right) \cdot \nabla F^{\prime}(r)
\end{aligned}
$$

Using (10)-(18), we get the desired relative entropy.

\subsection{Relaxation of the regularity on the test function $(r, V, W)$ :}

Let us recall the regularity on the global weak-solution of the degenerate compressible Navier-Stokes equations with linear degenerate viscosity $\mu(\varrho)=\mu \varrho$ and $\lambda(\varrho)=0$ and assuming the pressure law to be $p(\varrho)=a \varrho^{\gamma}$ :

$$
\begin{gathered}
\sqrt{\varrho} \nabla \mathbf{u} \in L^{2}\left(0, T ; L^{2}(\Omega)\right), \quad F(\varrho) \in L^{\infty}\left(0, T ; L^{1}(\Omega)\right), \\
\sqrt{\varrho \mathbf{w}} \in L^{\infty}\left(0, T ; L^{2}(\Omega)\right), \quad \sqrt{\varrho} \mathbf{v} \in L^{\infty}\left(0, T: L^{2}(\Omega)\right), \quad \sqrt{\frac{p^{\prime}(\varrho)}{\varrho}} \nabla \varrho \in L^{2}\left(0, T ; L^{2}(\Omega)\right)
\end{gathered}
$$


with the relation $\varrho F^{\prime}(\varrho)-F(\varrho)=p(\varrho)$ assuming that initially

$$
F\left(\varrho_{0}\right) \in L^{1}(\Omega), \quad \frac{m_{0}}{\sqrt{\varrho}_{0}} \in L^{2}(\Omega), \quad \frac{M_{0}}{\sqrt{\varrho}_{0}} \in L^{2}(\Omega),
$$

where $m_{0}=\varrho_{0} \mathbf{w}_{0}$ and $M_{0}=\varrho_{0} \mathbf{v}_{0}$ if $\varrho_{0} \neq 0$ and 0 if $\varrho_{0}=0$. Moreover we have the following estimates on the velocity due to the Mellet-Vasseur estimate

$$
\varrho \ln \left(1+|\mathbf{u}|^{2}\right)\left(1+|\mathbf{u}|^{2}\right) \in L^{\infty}\left(0, T ; L^{2}(\Omega)\right)
$$

if initially it is the case. We need to impose at least the weak regularity on the target $(r, V, W)$ and $0<c \leq$ $r<+\infty$ to define the left-hand side namely

$$
\begin{gathered}
\sqrt{r} \nabla U \in L^{2}\left(0, T ; L^{2}(\Omega)\right), \quad F(r) \in L^{\infty}\left(0, T ; L^{1}(\Omega)\right), \\
\sqrt{r} W \in L^{\infty}\left(0, T ; L^{2}(\Omega)\right), \quad \sqrt{r} V \in L^{\infty}\left(0, T: L^{2}(\Omega)\right), \quad \sqrt{\frac{p^{\prime}(r)}{r}} \nabla r \in L^{2}\left(0, T ; L^{2}(\Omega)\right) .
\end{gathered}
$$

Concerning the right-hand side to be well defined, we need the extra regularity

$$
\begin{gathered}
\left.\partial_{t} F^{\prime}(r) \in L^{1}\left(0, T ; L^{3 / 2}(\Omega)\right) \cap L^{\gamma /(\gamma-1)}(\Omega)\right), \quad \nabla F^{\prime}(r) \in L^{1}\left(0, T ; L^{3}(\Omega) \cap L^{2 \gamma /(\gamma-1)}(\Omega)\right) \\
\nabla V, \quad \nabla W \in L^{1}\left(0, T ; L^{\infty}(\Omega)\right) \\
\nabla r \in L^{\infty}\left(0, T ; L^{3}(\Omega)\right) .
\end{gathered}
$$

\section{Weak-Strong Uniqueness.}

Let us consider a $\kappa$-entropy solution $(\varrho, \mathbf{u})$ of $(2)$ and define the modified velocities $\mathbf{v}=\mathbf{u}+2 \kappa \mu \nabla \log \varrho$ and $\mathbf{w}=2 \mu \sqrt{\kappa(1-\kappa)} \nabla \log \varrho$. Moreover, we assume that $(r, W, V)$ satisfies the augmented system together with the regularity assumptions of the previous section. We further suppose that $W=2 \mu \sqrt{\kappa(1-\kappa)} \nabla \log r$. Then let us prove that $(\varrho, \mathbf{v}, \mathbf{w})=(r, V, W)$ that means weak-strong uniqueness property. This gives $(\varrho, \mathbf{u})=(r, U)$ with $U=V-\sqrt{\kappa} W / \sqrt{(1-\kappa)}$.

More precisely let us prove the following result

Theorem 2. Let $\Omega$ be a periodic box. Suppose that the pressure law satisfies $p(\varrho)=a \varrho^{\gamma}$ namely the power law pressure. Let $(\varrho, \mathbf{u})$ be a $\kappa$-entropy solution to the compressible Navier-Stokes system in the sense of the definition 1. Assume that there exists a strong solution of the compressible Navier-Stokes equations satisfying the positivity and regularity properties described previously in (3.1) and that $\log (r) \in L^{2}\left(0, T ; W^{1, \infty}(\Omega)\right) \cap L^{1}\left(0, T ; W^{2, \infty}(\Omega)\right)$. Then we have the weak-strong uniqueness result: $(\varrho, \mathbf{u})=(r, U)$.

Remark that we cannot test the equations satisfied by $V$ and $W$ by $V-\mathbf{v}$ and $W-\mathbf{w}$ because $\mathbf{v}$ and $\mathbf{w}$ are not controlled close to vacuum but $\sqrt{\varrho} \mathbf{v}$ and $\sqrt{\varrho} \mathbf{w}$ are well defined. Thus, let us take the equations satisfied by 
$V$ and $W$ and test them respectively against $\varrho(W-\mathbf{w}) / r$ and $\varrho(V-\mathbf{v}) / r$, we get

$$
\begin{aligned}
& \int_{0}^{\tau} \int_{\Omega} \varrho\left[\partial_{t} V \cdot(V-\mathbf{v})\right] \\
& +\int_{0}^{\tau} \int_{\Omega} \varrho\left(\left(\left(V-\sqrt{\frac{\kappa}{(1-\kappa)}} W\right) \cdot \nabla V\right) \cdot(V-\mathbf{v})\right) \\
& +\int_{0}^{\tau} \int_{\Omega} 2 \mu(1-\kappa) \varrho D(V): D(V-\mathbf{v})+\int_{0}^{\tau} \int_{\Omega} 2 \mu \kappa \varrho A(V): A(V-\mathbf{v}) \\
& -\int_{0}^{\tau} \int_{\Omega} 2 \mu \sqrt{\kappa(1-\kappa)} \varrho \nabla W: D(V-\mathbf{v})-\int_{0}^{\tau} \int_{\Omega} 2 \mu \sqrt{\kappa(1-\kappa)} \varrho \nabla W: A(V-\mathbf{v}) \\
& +\int_{0}^{\tau} \int_{\Omega} \frac{\varrho}{r} \nabla p(r) \cdot(V-\mathbf{v}) \\
& +\int_{0}^{\tau} \int_{\Omega} 2 \mu(1-\kappa) r D(V): \nabla\left(\frac{\varrho}{r}\right)(V-\mathbf{v})+\int_{0}^{\tau} \int_{\Omega} 2 \mu \kappa r A(V): \nabla\left(\frac{\varrho}{r}\right)(V-\mathbf{v}) \\
& -\int_{0}^{\tau} \int_{\Omega} 2 \mu \sqrt{\kappa(1-\kappa) r} r \nabla W: \nabla\left(\frac{\varrho}{r}\right)(V-\mathbf{v})=0
\end{aligned}
$$

and

$$
\begin{aligned}
& \int_{0}^{\tau} \int_{\Omega} \varrho\left[\partial_{t} W \cdot(W-\mathbf{w})\right]+\int_{0}^{\tau} \int_{\Omega} \varrho\left(\left(\left(V-\sqrt{\frac{\kappa}{(1-\kappa)}} W\right) \cdot \nabla W\right) \cdot(W-\mathbf{w})\right) \\
& +\int_{0}^{\tau} \int_{\Omega} 2 \mu \kappa \varrho \nabla(W): \nabla(W-\mathbf{w}) \\
& -\int_{0}^{\tau} \int_{\Omega} 2 \mu \sqrt{\kappa(1-\kappa)} \varrho D(V): \nabla(W-\mathbf{w})+\int_{0}^{\tau} \int_{\Omega} 2 \mu \sqrt{\kappa(1-\kappa)} \varrho A(V): \nabla(W-\mathbf{w}) \\
& +\int_{0}^{\tau} \int_{\Omega} 2 \mu \kappa r \nabla(W): \nabla\left(\frac{\varrho}{r}\right)(W-\mathbf{w})-\int_{0}^{\tau} \int_{\Omega} 2 \mu \sqrt{\kappa(1-\kappa)} r(\nabla V)^{t}: \nabla\left(\frac{\varrho}{r}\right)(W-\mathbf{w})=0 .
\end{aligned}
$$

Collecting the two previous equality, we get

$$
\begin{aligned}
& \int_{0}^{\tau} \int_{\Omega} \varrho\left[\partial_{t} V \cdot(V-\mathbf{v})\right]+\int_{0}^{\tau} \int_{\Omega} \varrho\left[\partial_{t} W \cdot(W-\mathbf{w})\right] \\
& +2 \mu \int_{0}^{\tau} \int_{\Omega} \varrho[D(\sqrt{(1-\kappa)} V)-\nabla(\sqrt{\kappa} W)]:[D(\sqrt{(1-\kappa)}(V-\mathbf{v}))-\nabla(\sqrt{\kappa}(W-\mathbf{w}))] \\
& +2 \mu \kappa \int_{0}^{\tau} \int_{\Omega} \varrho A(V): A(V-\mathbf{v})+2 \sqrt{\kappa(1-\kappa)} \mu \int_{0}^{\tau} \int_{\Omega} \varrho[A(W): A(\mathbf{v}-V)-A(\mathbf{w}-W): A(V)] \\
& =-\int_{0}^{\tau} \int_{\Omega} \varrho\left(\left(\left(V-\sqrt{\frac{\kappa}{(1-\kappa)}} W\right) \cdot \nabla V\right) \cdot(V-\mathbf{v})\right)-\int_{0}^{\tau} \int_{\Omega} \varrho\left(\left(\left(V-\sqrt{\frac{\kappa}{(1-\kappa)}} W\right) \cdot \nabla W\right) \cdot(W-\mathbf{w})\right) \\
& -\int_{0}^{\tau} \int_{\Omega} \frac{\varrho}{r} \nabla p(r) \cdot(V-\mathbf{v})-\int_{0}^{\tau} \int_{\Omega} 2 \mu(1-\kappa) r D(V): \nabla\left(\frac{\varrho}{r}\right)(V-\mathbf{v}) \\
& \left.-\int_{0}^{\tau} \int_{\Omega}^{\tau} 2 \mu(1-\kappa) r A(V): \nabla\left(\frac{\varrho}{r}\right)(V-\mathbf{v})+\int_{0}^{\tau} \int_{\Omega} 2 \mu(1-\kappa) r \nabla(W): \nabla\left(\frac{\varrho}{r}\right) V-\mathbf{v}\right) \\
& -\int_{0}^{\tau} \int_{\Omega}^{\tau} 2 \mu \kappa r \nabla(W): \nabla\left(\frac{\varrho}{r}\right)(W-\mathbf{w})+\int_{0}^{\tau} \int_{\Omega} 2 \mu \sqrt{\kappa(1-\kappa)} r(\nabla V)^{t}: \nabla\left(\frac{\varrho}{r}\right)(W-\mathbf{w})
\end{aligned}
$$


Now we can use this result to reformulate (9). We first remark that $F^{\prime \prime}(r)=p^{\prime}(r) / r$ and therefore we can write

$$
\begin{aligned}
& -\int_{0}^{\tau} \int_{\Omega} \frac{\varrho}{r} \nabla p(r) \cdot(V-\mathbf{v}) \\
& =-\int_{0}^{\tau} \int_{\Omega} \varrho \nabla F^{\prime}(r) \cdot\left[(V-\mathbf{v})-\sqrt{\frac{\kappa}{(1-\kappa)}}(W-\mathbf{w})\right]-\int_{0}^{\tau} \int_{\Omega} \frac{\varrho}{r} p^{\prime}(r) \nabla r \cdot \sqrt{\frac{\kappa}{(1-\kappa)}(W-\mathbf{w})}
\end{aligned}
$$

Thus, using the following identity

$$
p^{\prime}(\varrho) \nabla \varrho \cdot\left[2 \mu \frac{\nabla r}{r}-\frac{1}{\sqrt{(1-\kappa) \kappa}} W\right]+2 \mu \frac{\varrho}{r} p^{\prime}(r) \nabla r \cdot\left[\frac{\nabla r}{r}-\frac{\nabla \varrho}{\varrho}\right]-\frac{\varrho}{r} p^{\prime}(r) \nabla r \cdot \sqrt{\frac{\kappa}{(1-\kappa)}}(W-\mathbf{w})=0
$$

which is true if $W=2 \mu \sqrt{\kappa(1-\kappa)} \nabla \log r$ and $\mathbf{w}=2 \mu \sqrt{\kappa(1-\kappa)} \nabla \log \varrho$, we get

$$
\begin{aligned}
& E(\rho, v, w \mid r, V, W)(\tau)-E(\rho, v, w \mid r, V, W)(0) \\
& +2 \kappa \mu \int_{0}^{\tau} \int_{\Omega} \varrho|A(\mathbf{v}-V)|^{2}+2 \mu \int_{0}^{\tau} \int_{\Omega} \varrho|D(\sqrt{(1-\kappa)}(\mathbf{v}-V)-\sqrt{\kappa}(\mathbf{w}-W))|^{2} \\
& +2 \kappa \int_{0}^{\tau} \int_{\Omega}\left|\sqrt{\frac{p^{\prime}(\varrho)}{\varrho}} \nabla \varrho-\sqrt{\frac{p^{\prime}(r)}{r}} \nabla r\right|^{2} \\
& \left.\leq \int_{0}^{\tau} \int_{\Omega} \varrho\left(\left(\left(\mathbf{v}-\sqrt{\frac{\kappa}{(1-\kappa)}} \mathbf{w}\right)-\left(V-\sqrt{\frac{\kappa}{(1-\kappa)}} W\right)\right) \cdot \nabla V\right) \cdot(V-\mathbf{v})\right) \\
& \left.+\int_{0}^{\tau} \int_{\Omega} \varrho\left(\left(\left(\mathbf{v}-\sqrt{\frac{\kappa}{(1-\kappa)}} \mathbf{w}\right)-\left(V-\sqrt{\frac{\kappa}{(1-\kappa)}} W\right)\right) \cdot \nabla W\right) \cdot(W-\mathbf{w})\right) \\
& +\int_{0}^{\tau} \int_{\Omega}\left(\partial_{t} F^{\prime}(r)+\left(V-\sqrt{\frac{\kappa}{(1-\kappa)}} W\right) \cdot \nabla F^{\prime}(r)\right)(r-\varrho) \\
& +\int_{0}^{\tau} \int_{\Omega}(p(r)-p(\varrho)) \operatorname{div}\left(V-\sqrt{\frac{\kappa}{(1-\kappa)}} W\right) \\
& -\int_{0}^{\tau} \int_{\Omega}^{\tau} 2 \mu(1-\kappa) r D(V): \nabla\left(\frac{\varrho}{r}\right)(V-\mathbf{v}) \\
& -\int_{0}^{\tau} \int_{\Omega}^{\tau} 2 \mu(1-\kappa) r A(V): \nabla\left(\frac{\varrho}{r}\right)(V-\mathbf{v})+\int_{0}^{\tau} \int_{\Omega} 2 \mu(1-\kappa) r \nabla(W): \nabla\left(\frac{\varrho}{r}\right)(V-\mathbf{v}) \\
& -\int_{0}^{\tau} \int_{\Omega}^{\tau} 2 \mu \kappa r \nabla(W): \nabla\left(\frac{\varrho}{r}\right)(W-\mathbf{w})+\int_{0} 2 \mu \sqrt{\kappa(1-\kappa)} r(\nabla V)^{t}: \nabla\left(\frac{\varrho}{r}\right)(W-\mathbf{w})
\end{aligned}
$$

Note now that

$$
\left(\partial_{t} F^{\prime}(r)+\left(V-\sqrt{\frac{\kappa}{(1-\kappa)}} W\right) \cdot \nabla F^{\prime}(r)\right)(r-\varrho)=-\operatorname{div}\left(V-\sqrt{\frac{\kappa}{(1-\kappa)}} W\right)(\varrho-r) p^{\prime}(r)
$$

because

$$
F^{\prime \prime}(r)=\frac{p^{\prime}(r)}{r} \quad \text { and } \quad \partial_{t} r+\operatorname{div}\left(r\left(V-\sqrt{\frac{\kappa}{(1-\kappa)}} W\right)\right)=0
$$


Thus we conclude that

$$
\begin{aligned}
& E(\rho, v, w \mid r, V, W)(\tau)-E(\rho, v, w \mid r, V, W)(0) \\
& +2 \kappa \mu \int_{0}^{\tau} \int_{\Omega} \varrho|A(\mathbf{v}-V)|^{2}+2 \mu \int_{0}^{\tau} \int_{\Omega} \varrho|D(\sqrt{(1-\kappa)}(\mathbf{v}-V)-\sqrt{\kappa}(\mathbf{w}-W))|^{2} \\
& +2 \kappa \int_{0}^{\tau} \int_{\Omega} \varrho\left[p^{\prime}(\varrho) \nabla \log \varrho-p^{\prime}(r) \nabla \log r\right] \cdot[\nabla \log \varrho-\nabla \log r] \\
& \left.\leq \int_{0}^{\tau} \int_{\Omega} \varrho\left(\left(\left(\mathbf{v}-\sqrt{\frac{\kappa}{(1-\kappa)}} \mathbf{w}\right)-\left(V-\sqrt{\frac{\kappa}{(1-\kappa)}} W\right)\right) \cdot \nabla V\right) \cdot(V-\mathbf{v})\right) \\
& \left.+\int_{0}^{\tau} \int_{\Omega} \varrho\left(\left(\left(\mathbf{v}-\sqrt{\frac{\kappa}{(1-\kappa)}} \mathbf{w}\right)-\left(V-\sqrt{\frac{\kappa}{(1-\kappa)}} W\right)\right) \cdot \nabla W\right) \cdot(W-\mathbf{w})\right) \\
& +\int_{0}^{\tau} \int_{\Omega}\left(p(r)-p(\varrho)+p^{\prime}(r)(\varrho-r)\right) \operatorname{div}\left(V-\sqrt{\frac{\kappa}{(1-\kappa)}} W\right) \\
& -\int_{0}^{\tau} \int_{\Omega} 2 \mu(1-\kappa) r D(V): \nabla\left(\frac{\varrho}{r}\right)(V-\mathbf{v}) \\
& -\int_{0}^{\tau} \int_{\Omega}^{\tau} 2 \mu(1-\kappa) r A(V): \nabla\left(\frac{\varrho}{r}\right)(V-\mathbf{v})+\int_{0}^{\tau} \int_{\Omega} 2 \mu(1-\kappa) r \nabla(W): \nabla\left(\frac{\varrho}{r}\right)(V-\mathbf{v}) \\
& -\int_{0}^{\tau} \int_{\Omega} 2 \mu \kappa r \nabla(W): \nabla\left(\frac{\varrho}{r}\right)(W-\mathbf{w})+\int_{0}^{\tau} \int_{\Omega} 2 \mu \sqrt{\kappa(1-\kappa)} r(\nabla V)^{t}: \nabla\left(\frac{\varrho}{r}\right)(W-\mathbf{w}) .
\end{aligned}
$$

Note that

$$
\begin{aligned}
& \left.\mid \int_{\Omega} \varrho\left(\left(\left(\mathbf{v}-\sqrt{\frac{\kappa}{(1-\kappa)}} \mathbf{w}\right)-\left(V-\sqrt{\frac{\kappa}{(1-\kappa)}} W\right)\right) \cdot \nabla V\right) \cdot(V-\mathbf{v})\right) \\
& \left.+\int_{\Omega} \varrho\left(\left(\left(\mathbf{v}-\sqrt{\frac{\kappa}{(1-\kappa)}} \mathbf{w}\right)-\left(V-\sqrt{\frac{\kappa}{(1-\kappa)}} W\right)\right) \cdot \nabla W\right) \cdot(W-\mathbf{w})\right) \\
& +\int_{\Omega}\left(p(r)-p(\varrho)+p^{\prime}(r)(\varrho-r)\right) \operatorname{div}\left(V-\sqrt{\frac{\kappa}{(1-\kappa)}} W\right) \mid \\
& \leq c\left(\|\nabla V\|_{L^{\infty}(\Omega)}+\|\nabla W\|_{L^{\infty}(\Omega)}\right) E(\varrho, v, w \mid r, V, W)
\end{aligned}
$$

Finally recalling that $w=2 \sqrt{\kappa(1-\kappa)} \nabla \varrho / \varrho$, we remark that

$$
\nabla\left(\frac{\varrho}{r}\right)=\frac{\varrho}{r}[\nabla \log \varrho-\nabla \log r]=\frac{1}{2 \sqrt{\kappa(1-\kappa)}} \frac{\varrho}{r}(\mathbf{w}-W)
$$

Then we write

$$
\begin{aligned}
& {\left[\int_{\Omega} 2 \mu(1-\kappa) r D(V): \nabla\left(\frac{\varrho}{r}\right)(V-\mathbf{v})\right.} \\
& -\int_{\Omega} 2 \mu(1-\kappa) r A(V): \nabla\left(\frac{\varrho}{r}\right)(V-\mathbf{v})+\int_{\Omega} 2 \mu(1-\kappa) r \nabla(W): \nabla\left(\frac{\varrho}{r}\right)(V-\mathbf{v}) \\
& -\int_{\Omega} 2 \mu \kappa r \nabla(W): \nabla\left(\frac{\varrho}{r}\right)(W-\mathbf{w})+\int_{\Omega} 2 \mu \sqrt{\kappa(1-\kappa)} r(\nabla V)^{t}: \nabla\left(\frac{\varrho}{r}\right)(W-\mathbf{w}) \mid \\
& \leq c\left(\|\nabla V\|_{L^{\infty}(\Omega)}+\|\nabla W\|_{L^{\infty}(\Omega)}\right) E(\varrho, v, w \mid r, V, W)
\end{aligned}
$$


Let us now study the term in the left hand-side

$$
I_{1}=\int_{0}^{\tau} \int_{\Omega} \varrho\left[p^{\prime}(\varrho) \nabla \log \varrho-p^{\prime}(r) \nabla \log r\right] \cdot(\nabla \log \varrho-\nabla \log r]
$$

which has a priori no sign. We can write it as

$$
I=\int_{0}^{\tau} \int_{\Omega} \varrho p^{\prime}(\varrho)|\nabla \log \varrho-\nabla \log r|^{2}+\int_{0}^{\tau} \int_{\Omega} \varrho\left(p^{\prime}(\varrho)-p^{\prime}(r)\right) \nabla \log r \cdot(\nabla \log \varrho-\nabla \log r)=I_{1}+I_{2}
$$

The first term $I_{1}$ is positive, there it remains to bound the second term $I_{2}$. Let us recall that we consider the case $p(\varrho)=a \varrho^{\gamma}$. Then, we have to consider the quantity

$$
I=\int_{0}^{\tau} \int_{\Omega} \varrho\left[\varrho^{\gamma-1} \nabla \log \varrho-r^{\gamma-1} \nabla \log r\right] \cdot(\nabla \log \varrho-\nabla \log r]
$$

which may be written

$$
I=\int_{0}^{\tau} \int_{\Omega} \varrho^{\gamma}|\nabla \log \varrho-\nabla \log r|^{2}+\int_{0}^{\tau} \int_{\Omega}\left[\varrho^{\gamma}-\varrho r^{\gamma-1}\right] \nabla \log r \cdot[\nabla \log \varrho-\nabla \log r]=I_{1}+I_{2}
$$

The first quantity $I_{1}$ is positive. It remains to control $I_{2}$. It is important now to observe the following identity Let us notice that

$$
\begin{aligned}
\varrho\left[p^{\prime}(\varrho)-p^{\prime}(r)\right] \nabla \log r \cdot & {[\nabla \log \varrho-\nabla \log r]=\nabla\left[p(\varrho)-p(r)-p^{\prime}(r)(\varrho-r)\right] \cdot \nabla \log r } \\
- & {\left[\varrho\left(p^{\prime}(\varrho)-p^{\prime}(r)\right)-p^{\prime \prime}(r)(\varrho-r) r\right]|\nabla \log r|^{2} }
\end{aligned}
$$

Thus

$$
\begin{aligned}
I_{2} \leq & \frac{1}{\gamma}\left[\int_{0}^{\tau} \int_{\Omega}\left|\left(p(\varrho)-p(r)-p^{\prime}(r)(\varrho-r)\right)\right||\Delta \log r|\right. \\
& \quad+\int_{0}^{\tau} \int_{\Omega} \mid\left(\varrho\left(p^{\prime}(\varrho)-p^{\prime}(r)\right)-\left.p^{\prime \prime}(r)(\varrho-r) r|| \nabla \log r\right|^{2}\right] \\
& \leq J_{1}+J_{2} .
\end{aligned}
$$

The key-point is now to recall that

$$
\left.p(\varrho)-p(r)-p^{\prime}(r)(\varrho-r)\right) \approx F(\varrho \mid r)
$$

where $F(\varrho \mid r)=F(\varrho)-F(r)-F^{\prime}(r)(\varrho-r)$ by definition and that it is possible to prove (see Lemma 2.2. [1]) that

$$
\varrho\left(p^{\prime}(\varrho)-p^{\prime}(r)\right)-p^{\prime \prime}(r)(\varrho-r) r \approx F(\varrho \mid r)
$$

Thus

$$
I_{2} \leq C \int_{0}^{\tau}\left[\left(\|\Delta \log r\|_{L^{\infty}(\Omega)}+\|\nabla \log r\|_{L^{\infty}(\Omega)}^{2}\right) E(\varrho, \mathbf{v}, \mathbf{w} \mid r, V, W)\right] .
$$

This ends the proof of weak-strong uniqueness using Gronwall Lemma asking $\log r$ to be bounded in $L^{2}\left(0, T ; W^{1, \infty}(\Omega)\right) \cap$ $L^{1}\left(0, T ; W^{2, \infty}(\Omega)\right)$. 


\section{Convergence to a Dissipative solution of COMPressible Euler equations.}

Let us recall the definition of a dissipative solution of compressible Euler equations. Such concept has been introduced by P.-L. Lions in the incompressible setting: see for instance [16]. The reader is referred to $[9],[20],[1]$ for the extension to the compressible framework.

Definition. The pair $(\bar{\varrho}, \bar{u})$ is a dissipative solution of the compressible Euler equations if and only if $(\bar{\varrho}, \bar{u})$ satisfies the relative energy inequality

$$
\begin{gathered}
E(\bar{\varrho}, \bar{u}, 0 \mid r, U, 0)(t) \leq E(\bar{\varrho}, \bar{u}, 0 \mid r, U, 0)(0) \exp \left[c_{0}(r) \int_{0}^{t}\|\operatorname{div} U(\tau)\|_{L^{\infty}(\Omega)} d \tau\right] \\
+\int_{0}^{t} \exp \left[c_{0}(r) \int_{s}^{t}\|\operatorname{div} U(\tau)\|_{L^{\infty}(\Omega)}\right] \int_{\Omega} \varrho E(r, U) \cdot(U-\bar{u}) d x d s
\end{gathered}
$$

for all smooth test functions $(r, U)$ defined on $[0, T] \times \bar{\Omega}$ so that $r$ is bounded above and below away from zero and $(r, U)$ solves

$$
\begin{aligned}
& \partial_{t} r+\operatorname{div}(r U)=0 \\
& \partial_{t} U+U \cdot \nabla U+\nabla F^{\prime}(r)=E(r, U)
\end{aligned}
$$

for some residual $E(r, U)$.

We prove the following result.

Theorem 3. Let $\left(\varrho_{\varepsilon}, \mathbf{u}_{\varepsilon}\right)$ be any finite $\kappa$-entropy solution to the viscous compressible Navier-Stokes equations in the periodic setting namely $\mu(\varrho)=\varepsilon \varrho, \lambda(\varrho)=0$ and $p(\varrho)=a \varrho^{\gamma}$. Then, any weak limit $(\varrho, \bar{u})$ of $\left(\varrho_{\varepsilon}, \mathbf{u}_{\varepsilon}\right)$, when $\varepsilon \rightarrow 0$, in the sense

$$
\begin{aligned}
& \varrho_{\varepsilon} \rightarrow \bar{\varrho} \text { weakly } \star \text { in } L^{\infty}\left(0, T ; L^{\gamma}(\Omega)\right), \\
& \sqrt{\varrho_{\varepsilon}} \mathbf{v}_{\varepsilon} \rightarrow \sqrt{\bar{\varrho}} \bar{u} \text { weakly } \star \text { in } L^{\infty}\left(0, T ; L^{2}(\Omega)\right) \\
& \sqrt{\varrho_{\varepsilon}} \mathbf{w}_{\varepsilon} \rightarrow 0 \text { weakly } \star \text { in } L^{\infty}\left(0, T ; L^{2}(\Omega)\right)
\end{aligned}
$$

with $\mathbf{v}_{\varepsilon}=\mathbf{u}_{\varepsilon}+2 \varepsilon \kappa \nabla \log \varrho_{\varepsilon}$ and $\mathbf{w}_{\varepsilon}=2 \varepsilon \sqrt{\kappa(1-\kappa)} \nabla \log \varrho_{\varepsilon}$ as $\varepsilon$ tends to zero, is a dissipative solution to the compressible compressible Euler equations. 
Proof. Let us consider the $\kappa$ entropy solution $\left(\varrho_{\varepsilon}, \mathbf{u}_{\varepsilon}\right)$ of system $(2)$ with $\mu(\varrho)=\varepsilon \varrho$ and $\lambda(\varrho)=0$. It has been shown that it satisfies the so-called relative entropy inequality

$$
\begin{aligned}
& E\left(\varrho_{\varepsilon}, \mathbf{v}_{\varepsilon}, \mathbf{w}_{\varepsilon} \mid r, V, W\right)(\tau)-E\left(\varrho_{\varepsilon}, \mathbf{v}_{\varepsilon}, \mathbf{w}_{\varepsilon} \mid r, V, W\right)(0) \\
& +2 \kappa \varepsilon \int_{0}^{\tau} \int_{\Omega} \varrho_{\varepsilon}\left|A\left(\mathbf{v}_{\varepsilon}-V\right)\right|^{2}+2 \varepsilon \int_{0}^{\tau} \int_{\Omega} \varrho_{\varepsilon}\left|D\left(\sqrt{(1-\kappa)}\left(\mathbf{v}_{\varepsilon}-V\right)-\sqrt{\kappa}\left(\mathbf{w}_{\varepsilon}-W\right)\right)\right|^{2} \\
& +2 \kappa \varepsilon \int_{0}^{\tau} \int_{\Omega} \varrho_{\varepsilon}\left[p^{\prime}\left(\varrho_{\varepsilon}\right) \nabla \log \varrho_{\varepsilon}-p^{\prime}(r) \nabla \log r\right] \cdot\left[\nabla \log \varrho_{\varepsilon}-\nabla \log r\right] \\
& \leq \int_{0}^{\tau} \int_{\Omega} \varrho_{\varepsilon}\left(\left(\left(\mathbf{v}_{\varepsilon}-\sqrt{\frac{\kappa}{(1-\kappa)}} \mathbf{w}_{\varepsilon}\right) \cdot \nabla W\right) \cdot\left(W-\mathbf{w}_{\varepsilon}\right)+\left(\left(\mathbf{v}_{\varepsilon}-\sqrt{\frac{\kappa}{(1-\kappa)}} \mathbf{w}\right) \cdot \nabla V\right) \cdot\left(V-\mathbf{v}_{\varepsilon}\right)\right) \\
& +\int_{0}^{\tau} \int_{\Omega} \varrho_{\varepsilon}\left(\partial_{t} W \cdot\left(W-\mathbf{w}_{\varepsilon}\right)+\partial_{t} V \cdot\left(V-\mathbf{v}_{\varepsilon}\right)\right) \\
& +\int_{0}^{\tau} \int_{\Omega} \partial_{t} F^{\prime}(r)\left(r-\varrho_{\varepsilon}\right)-\int_{0}^{\tau} \int_{\Omega} \nabla F^{\prime}(r) \cdot\left[\varrho_{\varepsilon}\left(\mathbf{v}_{\varepsilon}-\sqrt{\frac{\kappa}{(1-\kappa)}} \mathbf{w}_{\varepsilon}\right)-r\left(V-\sqrt{\frac{\kappa}{(1-\kappa)}} W\right)\right] \\
& +\int_{0}^{\tau} \int_{\Omega}\left(p(r)-p\left(\varrho_{\varepsilon}\right)\right) \operatorname{div}\left(V-\sqrt{\frac{\kappa}{(1-\kappa)}} W\right) \\
& -\kappa \int_{0}^{\tau} \int_{\Omega} p^{\prime}\left(\varrho_{\varepsilon}\right) \nabla \varrho_{\varepsilon} \cdot\left[2 \varepsilon \frac{\nabla r}{r}-\frac{1}{\sqrt{(1-\kappa) \kappa}} W\right] \\
& \left.+2 \varepsilon \int_{0}^{\tau} \int_{\Omega} \varrho_{\varepsilon}(D(\sqrt{(1-\kappa)} V)-\nabla(\sqrt{\kappa} W))\right):\left(D\left(\sqrt{(1-\kappa)}\left(V-\mathbf{v}_{\varepsilon}\right)\right)-\nabla\left(\sqrt{\kappa}\left(W-\mathbf{w}_{\varepsilon}\right)\right)\right) \\
& +2 \kappa \varepsilon \int_{0}^{\tau} \int_{\Omega} \varrho_{\varepsilon} A(V): A\left(V-\mathbf{v}_{\varepsilon}\right)+2 \kappa \varepsilon \int_{0}^{\tau} \int_{\Omega} \frac{\varrho_{\varepsilon}}{r} p^{\prime}(r) \nabla r \cdot\left(\frac{\nabla r}{r}-\frac{\nabla \varrho_{\varepsilon}}{\varrho_{\varepsilon}}\right) \\
& +2 \sqrt{\kappa(1-\kappa)} \varepsilon \int_{0}^{\tau} \int_{\Omega} \varrho_{\varepsilon}\left[A(W): A\left(\mathbf{v}_{\varepsilon}-V\right)-A\left(\mathbf{w}_{\varepsilon}-W\right): A(V)\right]
\end{aligned}
$$

Let us now consider $(r, U)$ as in the definition of dissipative solution for compressible Euler equations and define $\left(r, V_{\varepsilon}, W_{\varepsilon}\right)$ through the relations $W_{\varepsilon}=2 \varepsilon \sqrt{\kappa(1-\kappa)} \nabla r / r$ and $V_{\varepsilon}=U+2 \varepsilon \kappa \nabla r / r$. Therefore

$$
\begin{aligned}
& \partial_{t} r+\operatorname{div}(r U)=0, \\
& \partial_{t} V_{\varepsilon}+U \cdot \nabla V_{\varepsilon}+\nabla F^{\prime}(r)=E(r, U)-\frac{2 \varepsilon}{r} \operatorname{div}\left(r(\nabla U)^{t}\right)=E_{1}^{\varepsilon}(r, U) \\
& \partial_{t} W_{\varepsilon}+U \cdot \nabla W_{\varepsilon}=-\frac{2 \sqrt{\kappa(1-\kappa) \varepsilon}}{r} \operatorname{div}\left(r(\nabla U)^{t}\right)=E_{2}^{\varepsilon}(r, U)
\end{aligned}
$$

Observe now that

$$
p\left(\varrho_{\varepsilon}\right)-p(r)=\varrho\left(F^{\prime}\left(\varrho_{\varepsilon}\right)-F^{\prime}(r)\right)-F\left(\varrho_{\varepsilon} \mid r\right)
$$

where

$$
F\left(\varrho_{\varepsilon} \mid r\right)=F\left(\varrho_{\varepsilon}\right)-F(r)-F^{\prime}(r)\left(\varrho_{\varepsilon}-r\right)
$$


Then after some calculations we can prove that the relative entropy may be written as

$$
\begin{aligned}
& E\left(\varrho_{\varepsilon}, \mathbf{v}_{\varepsilon}, \mathbf{w}_{\varepsilon} \mid r, V_{\varepsilon}, W_{\varepsilon}\right)(\tau)-E\left(\varrho_{\varepsilon}, \mathbf{v}_{\varepsilon}, \mathbf{w}_{\varepsilon} \mid r, V_{\varepsilon}, W_{\varepsilon}\right)(0) \\
& +2 \kappa \varepsilon \int_{0}^{\tau} \int_{\Omega} \varrho_{\varepsilon}\left|A\left(\mathbf{v}_{\varepsilon}-V_{\varepsilon}\right)\right|^{2}+2 \varepsilon \int_{0}^{\tau} \int_{\Omega} \varrho_{\varepsilon}\left|D\left(\sqrt{(1-\kappa)}\left(\mathbf{v}^{\varepsilon}-V^{\varepsilon}\right)-\sqrt{\kappa}\left(\mathbf{w}^{\varepsilon}-W^{\varepsilon}\right)\right)\right|^{2} \\
& +2 \kappa \varepsilon \int_{0}^{\tau} \int_{\Omega} \varrho^{\varepsilon}\left[p^{\prime}\left(\varrho_{\varepsilon}\right) \nabla \log \varrho_{\varepsilon}-p^{\prime}(r) \nabla \log r\right] \cdot\left[\nabla \log \varrho_{\varepsilon}-\nabla \log r\right] \\
& \leq \int_{0}^{\tau} \int_{\Omega} \varrho_{\varepsilon}\left(E_{1}^{\varepsilon}(r, U) \cdot\left(V_{\varepsilon}-\mathbf{v}_{\varepsilon}\right)+E_{2}^{\varepsilon}(r, U) \cdot\left(W_{\varepsilon}-\mathbf{w}_{\varepsilon}\right)\right) \\
& \left.-\int_{0}^{\tau} \int_{\Omega}\left(\varrho_{\varepsilon}\left(F^{\prime}\left(\varrho_{\varepsilon}\right)-F^{\prime}(r)\right)-r\left(\varrho_{\varepsilon}-r\right) F^{\prime \prime}(r)-F\left(\varrho_{\varepsilon} \mid r\right)\right)\right) \operatorname{div} U \\
& \left.+2 \varepsilon \int_{0}^{\tau} \int_{\Omega} \varrho_{\varepsilon}\left(D\left(\sqrt{(1-\kappa)} V_{\varepsilon}\right)-\nabla\left(\sqrt{\kappa} W_{\varepsilon}\right)\right)\right):\left(D\left(\sqrt{(1-\kappa)}\left(V_{\varepsilon}-\mathbf{v}_{\varepsilon}\right)\right)-\nabla\left(\sqrt{\kappa}\left(W_{\varepsilon}-\mathbf{w}_{\varepsilon}\right)\right)\right) \\
& +2 \kappa \varepsilon \int_{0}^{\tau} \int_{\Omega} \varrho_{\varepsilon} A\left(V_{\varepsilon}\right): A\left(V_{\varepsilon}-\mathbf{v}_{\varepsilon}\right)
\end{aligned}
$$

Now we use as in [1] that

$$
\left.\varrho_{\varepsilon}\left(F^{\prime}\left(\varrho_{\varepsilon}\right)-F^{\prime}(r)\right)-r\left(\varrho_{\varepsilon}-r\right) F^{\prime \prime}(r)\right) \approx F\left(\varrho_{\varepsilon} \mid r\right)
$$

thus after some Cauchy-Schwarz inequalities, we get

$$
\begin{aligned}
& E\left(\rho_{\varepsilon}, \mathbf{v}_{\varepsilon}, \mathbf{w}_{\varepsilon} \mid r, V_{\varepsilon}, W_{\varepsilon}\right)(\tau)-E\left(\rho_{\varepsilon}, \mathbf{v}_{\varepsilon}, \mathbf{w}_{\varepsilon} \mid r, V_{\varepsilon}, W_{\varepsilon}\right)(0) \\
& +2 \kappa \varepsilon \int_{0}^{\tau} \int_{\Omega} \varrho_{\varepsilon}\left|A\left(\mathbf{v}_{\varepsilon}-V_{\varepsilon}\right)\right|^{2}+2 \varepsilon \int_{0}^{\tau} \int_{\Omega} \varrho_{\varepsilon}\left|D\left(\sqrt{(1-\kappa)}\left(\mathbf{v}_{\varepsilon}-V_{\varepsilon}\right)-\sqrt{\kappa}\left(\mathbf{w}_{\varepsilon}-W_{\varepsilon}\right)\right)\right|^{2} \\
& +2 \kappa \varepsilon \int_{0}^{\tau} \int_{\Omega} \varrho_{\varepsilon}\left[p^{\prime}\left(\varrho_{\varepsilon}\right) \nabla \log \varrho_{\varepsilon}-p^{\prime}(r) \nabla \log r\right] \cdot\left[\nabla \log \varrho_{\varepsilon}-\nabla \log r\right] \\
& \leq \int_{0}^{\tau} \int_{\Omega} \varrho_{\varepsilon}\left(E_{1}^{\varepsilon}(r, U) \cdot\left(V_{\varepsilon}-\mathbf{v}_{\varepsilon}\right)+E_{2}^{\varepsilon}(r, U) \cdot\left(W_{\varepsilon}-\mathbf{w}_{\varepsilon}\right)\right) \\
& +\int_{0}^{\tau}\left[c(r)\|\operatorname{div} U\|_{L^{\infty}(\Omega)} F\left(\varrho_{\varepsilon} \mid r\right)\right] \\
& \left.+2 \varepsilon \int_{0}^{\tau} \int_{\Omega} \varrho_{\varepsilon}\left(D\left(\sqrt{(1-\kappa)} V_{\varepsilon}\right)-\nabla\left(\sqrt{\kappa} W_{\varepsilon}\right)\right)\right):\left(D\left(\sqrt{(1-\kappa)}\left(V_{\varepsilon}-\mathbf{v}_{\varepsilon}\right)\right)-\nabla\left(\sqrt{\kappa}\left(W_{\varepsilon}-\mathbf{w}_{\varepsilon}\right)\right)\right) \\
& +2 \kappa \varepsilon \int_{0}^{\tau} \int_{\Omega} \varrho_{\varepsilon} A\left(V_{\varepsilon}\right): A\left(V_{\varepsilon}-\mathbf{v}_{\varepsilon}\right)
\end{aligned}
$$

. It remains now to deals with the pressure term in the left-hand side

$$
I=\int_{0}^{\tau} \int_{\Omega} \varrho_{\varepsilon}\left[p^{\prime}\left(\varrho_{\varepsilon}\right) \nabla \log \varrho_{\varepsilon}-p^{\prime}(r) \nabla \log r\right] \cdot\left[\nabla \log \varrho_{\varepsilon}-\nabla \log r\right]
$$

which as a-priori no sign. We can recall what as been done in the weak-strong uniqueness part namely

$$
I=\int_{0}^{\tau} \int_{\Omega} \varrho_{\varepsilon} p^{\prime}\left(\varrho_{\varepsilon}\right)\left|\nabla \log \varrho_{\varepsilon}-\nabla \log r\right|^{2}+\int_{0}^{\tau} \int_{\Omega} \varrho_{\varepsilon}\left(p^{\prime}\left(\varrho_{\varepsilon}\right)-p^{\prime}(r)\right) \nabla \log r \cdot\left(\nabla \log \varrho_{\varepsilon}-\nabla \log r\right)=I_{1}+I_{2}
$$

The first term $I_{1}$ is positive, there it remains to bound the second term $I_{2}$. Everything work as for the weakstrong uniqueness for $p(\varrho)=a \varrho^{\gamma}$ and $\mu(\varrho)=\mu \varrho, \lambda(\varrho)=0$ to control the term linked to the pressure namely $I_{2}$ 
. More precisely we can prove that

$$
\begin{aligned}
& E\left(\varrho_{\varepsilon}, \mathbf{v}_{\varepsilon}, \mathbf{w}_{\varepsilon} \mid r, V_{\varepsilon}, W_{\varepsilon}\right)(\tau) \\
& \leq E\left(\varrho_{\varepsilon}, \mathbf{v}_{\varepsilon}, \mathbf{w}_{\varepsilon} \mid r, V_{\varepsilon}, W_{\varepsilon}\right)(0) \\
& \quad \exp \left(c(r) \int_{0}^{t}\|\operatorname{div} U(\tau)\|_{L^{\infty}(\Omega}+2 \kappa \varepsilon\left(\|\nabla \log r(\tau)\|_{L^{\infty}(\Omega)}^{2}+\mid \Delta \log r(\tau) \|_{L^{\infty}(\Omega)}\right)\right) \\
& +\int_{0}^{t} \exp \left(c(r) \int_{s}^{t}\left(\|\operatorname{div} U(\tau)\|_{L^{\infty}(\Omega}+2 \kappa \varepsilon\left(\|\nabla \log r(\tau)\|_{L^{\infty}(\Omega)}^{2}+\mid \Delta \log r(\tau) \|_{L^{\infty}(\Omega)}\right)\right) d \tau\right) \\
& \int_{\Omega}\left[\left(\varrho^{\varepsilon}\left(E_{1}^{\varepsilon}(r, U) \cdot\left(V_{\varepsilon}-\mathbf{v}_{\varepsilon}\right)+E_{2}^{\varepsilon}(r, U) \cdot\left(W_{\varepsilon}-\mathbf{w}_{\varepsilon}\right)\right)\right)+C_{0} \varepsilon \varrho_{\varepsilon}|\nabla U|^{2}\right]
\end{aligned}
$$

This ends the proof letting $\varepsilon$ tend to zero.

\section{Convergence to the Strong solution of incompressible Euler equations in THE WELL-PREPARED CASE.}

In this part, we want to prove that our augmented formulation is well adapted to the low Mach number and inviscid limit together. This will help in a forthcoming paper, see [6], to justify the intuitive scheme for incompressible Euler System already defined in [13]: Remark that $\kappa$ may play the role of $h$ in [13] which is the mesh size. We will choose as pressure state $p(\rho)=a \rho^{\gamma}$. This extends to the density dependent case, the method and result obtained by E. FEIREISL et. al. in the constant viscosity case, see [9] and [18] and references cited therein. It would remain to consider the ill prepared case to end up the complete asymptotic analysis but it is not the objective of this proceeding. We also assume that the initial density converge to a constant taken as 1 namely $\left(\rho_{0}^{\varepsilon}-1\right) / \varepsilon^{2} \rightarrow 0$ in $L^{2}(\Omega)$, the initial velocity converging to an incompressible velocity $U_{0}$ in $L^{2}(\Omega)$. We start with the augmented system

$$
\left\{\begin{array}{c}
\partial_{t} \varrho+\operatorname{div}(\varrho(\mathbf{v}-2 \kappa \mu \nabla \log \varrho))=0, \\
\partial_{t}(\varrho \mathbf{v})+\operatorname{div}(\varrho \mathbf{v} \otimes(\mathbf{v}-2 \kappa \mu \nabla \log \varrho))+\frac{1}{\varepsilon^{2}} \nabla p(\rho)=\operatorname{div}(2 \mu \rho(1-\kappa) D(\mathbf{v}))+\operatorname{div}(2 \kappa \mu \rho A(\mathbf{v})) \\
\quad-\operatorname{div}(2 \sqrt{\kappa(1-\kappa)} \mu \rho \nabla \mathbf{w}), \\
\partial_{t}(\varrho \mathbf{w})+\operatorname{div}(\varrho \mathbf{w} \otimes(\mathbf{v}-2 \kappa \mu \nabla \log \varrho))=\operatorname{div}(2 \kappa \mu \rho \nabla \mathbf{w})-\operatorname{div}\left(2 \sqrt{\kappa(1-\kappa)} \mu \rho \nabla \mathbf{v}^{T}\right) .
\end{array}\right.
$$

We want to pass to the limit with respect to $\varepsilon$ and $\mu$ for some relation between the two. The entropy reads

$$
\begin{aligned}
& \sup _{\tau \in[0, t]}\left[\int_{\Omega} \varrho\left(\frac{|\mathbf{v}|^{2}}{2}+\frac{|\mathbf{w}|^{2}}{2}\right)(\tau) \mathrm{d} x+\int_{\Omega} F(\varrho)(\tau) \mathrm{d} x\right] \\
& +2 \mu \int_{0}^{t} \int_{\Omega} \varrho\left(\kappa|A(\mathbf{v})|^{2}+|D(\sqrt{1-\kappa} \mathbf{v})-\nabla(\sqrt{\kappa} \mathbf{w})|^{2}\right) \\
& \quad+2 \frac{\kappa \mu}{\varepsilon^{2}} \int_{0}^{t} \int_{\Omega} \frac{p^{\prime}(\varrho)}{\varrho}|\nabla \varrho|^{2} d x \mathrm{~d} x \leq \int_{\Omega} \varrho\left(\frac{|\mathbf{v}|^{2}}{2}+\frac{|\mathbf{w}|^{2}}{2}\right)(0) \mathrm{d} x+\int_{\Omega} F(\varrho)(0) \mathrm{d} x
\end{aligned}
$$

Let us choose $r=1, V=U$ the strong solution of the incompressible Euler Equation

$$
\partial_{t} U+U \cdot \nabla U+\nabla \Pi=0, \quad \operatorname{div} U=0
$$


and $W=0$. Recalling that

$$
E(\rho, \mathbf{v}, \mathbf{w} \mid 1, U, 0)=\frac{1}{2} \int_{\Omega} \varrho\left(|\mathbf{w}|^{2}+|\mathbf{v}-U|^{2}\right)+\frac{a}{\varepsilon^{2}(\gamma-1)} \int_{\Omega}\left(\varrho^{\gamma}-1-\gamma(\varrho-1)\right)
$$

the relative entropy reads

$$
\begin{aligned}
& E(\rho, v, w \mid 1, U, 0)(\tau)-E(\rho, v, w \mid 1, U, 0)(0) \\
& +2 \kappa \mu \int_{0}^{\tau} \int_{\Omega} \varrho|A(\mathbf{v}-U)|^{2}+2 \mu \int_{0}^{\tau} \int_{\Omega} \varrho|D(\sqrt{(1-\kappa)}(\mathbf{v}-U)-\sqrt{\kappa} \mathbf{w})|^{2} \\
& +2 \frac{\kappa \mu}{\varepsilon^{2}} \int_{0}^{\tau} \int_{\Omega} \varrho p^{\prime}(\varrho)|\nabla \log \varrho|^{2} \\
& \left.\leq \int_{0}^{\tau} \int_{\Omega} \varrho\left(\left((\mathbf{v}-U)-\sqrt{\frac{\kappa}{(1-\kappa)}} \mathbf{w}\right) \cdot \nabla U\right) \cdot(U-\mathbf{v})\right) \\
& +\int_{0}^{\tau} \int_{\Omega}^{\tau} \varrho\left(\partial_{t} U \cdot(U-\mathbf{v})\right) \\
& \left.\left.+2 \mu \int_{0}^{\tau} \int_{\Omega} \varrho(D(\sqrt{(1-\kappa)} U))\right)\right):(D(\sqrt{(1-\kappa)}(U-\mathbf{v}))+\nabla(\sqrt{\kappa} \mathbf{w})) \\
& +2 \kappa \mu \int_{0}^{\tau} \int_{\Omega} \varrho A(U): A(U-\mathbf{v}) .
\end{aligned}
$$

Playing with the regularity of $(U, \Pi)$ solution of the incompressible Euler equations, we can get as in the other parts a differential inequality allowing to conclude of the convergence when $\varepsilon \rightarrow 0$. Note that if we choose $\kappa \mu=O\left(\varepsilon^{2}\right)$, we get through the pressure term a convergence in $L^{2}\left(0, T ; H^{1}(\Omega)\right)$ norm of $\sqrt{\rho p^{\prime}(\rho)} \nabla \rho^{\varepsilon}$ to 1 .

\section{REFERENCES}

[1] C. Bardos, T. Nguyen. Remarks on the inviscid limit for the compressible flows. Contemporary Mathematics, AMS, To appear (2016).

[2] S. Benzoni-Gavage, R. Danchin, S. Descombes On the well-posedness for the Euler-Korteweg model in several space dimensions Indiana University Mathematics Journal, 56, no 4, (2007), 1499-1579.

[3] D. Bresch, B. Desjardins. Quelques modèles diffusifs capillaires de type Korteweg, C. R. Acad. Sci. Paris, section mécanique, 332, no. 11, (2004), 881-886.

[4] D. Bresch, B. Desjardins, C.K. Lin. On some compressible fluid models: Korteweg, lubrication and shallow water systems. Commun. Part. Diff. Eqs., Vol. 28, (2003), 1009-1037.

[5] D. Bresch, B. Desjardins, E. Zatorska. Two-velocity hydrodynamics in fluid mechanics: Part II. Existence of global $\kappa$ entropy solutions to compressible Navier-Stokes systems with degenerate viscosities. J. Math. Pures Appl., 104, Issue 4, (2015), 801-836.

[6] D. Bresch, P. Noble, J.-P. Vila, P. Villedieu. Numerical Schemes for some extended formulations of compressible NavierStokes equations. In preparation (2015).

[7] D. Bresch, P. Noble, J.-P. Vila. Relative entropy for compressible Navier-Stokes equations with density-dependent viscosities and applications. C. R. Acad. Sciences: Section Mathématiques. Volume 354, Issue 1, (2016), 45-49.

[8] C. M. Dafermos. The second law of thermodynamics and stability Arch. Rational Mech. Anal. 70(2):167-179, (1979).

[9] E. Feireisl. Relative entropies, dissipative solutions, and singular limits of complete fluid systems. Hyperbolic Problems: Theory, Numerics, Applications,, edited by: Fabio Ancona, Alberto Bressan, Pierangelo Marcati, Andrea Marson, AIMS on Applied Mathematics, vol. 8, AIMS, Sringfield, 2014, 11-28.

[10] E. Feireisl, B.J. Jin, A. Novotny. Relative entropies, suitable weak solutions and weak-strong uniqueness for the compressible Navier-Stokes system. J. Math. Fluid Mech, 14 (4):717-730, 2012.

[11] E. Feireisl, A. Novotny. Singular Limits in Thermodynamics of Viscous Fluids. Birkhäuser Verlag, (2009).

[12] Th. Gallouët, R. Herbin, D. Maltese, A. Novotny. Error estimates for a numerical approximation to the compressible barotropic Navier-Stokes equations.

[13] N. Grenier, J.-P. Vila, P. Villedieu. An accurate low-Mach scheme for a compressible two-fluid model applied to free-surface flows. J. Comput. Phys. 252, (2013), 1-19. 
[14] B. HAspot. Weak-Strong uniqueness for compressible Navier-Stokes system with degenerate viscosity coefficient and vacuum in one dimension. Submitted (2014).

[15] J. Li, Z. Xin. Global Existence of Weak Solutions to the Barotropic Compressible Navier-Stokes Flows with Degenerate Viscosities. Arxiv (2015): arXiv:1504.06826v2

[16] P.-L. Lions. Mathematical topics in fluid mechanics. Vol. 1 incompressible models. Oxford Science publication, (2006).

[17] A. Mellet, A. VAsseur. Existence and Uniqueness of Global Strong Solutions for One-Dimensional Compressible Navier?Stokes Equations. SIAM J. Math. Anal. 39 (4) (2008) 1344-1365.

[18] A. Novotny. Lecture Notes on Navier-Stokes-Fourier system. Panorama et synthèses, to appear SMF (2015).

[19] B. Perthame, P.E. Souganidis. Dissipative and entropy solutions to non-isotropic degenerate parabolic balance laws. Arch. Rational Mech Anal., (2003), 359-370.

[20] F. Sueur. On the inviscid limit for the compressible Navier-Stokes system in an impermeable bounded domain. J. Math. Fluid Mech. 16, (2014), no.1, 163-178.

[21] C. Christoforou, A.E. Tzavaras. Relative entropy for hyperbolic-parabolic systems and application to the constitutive theory of thermoviscoelasticity: arXiv:1603.08176.

[22] A. VAsseur, C. YU. Existence of global weak solutions for 3D degenerate compressible Navier-Stokes equations. Inventiones $1-40,(2016)$

[23] H.-T. YAu. Relative entropy and hydrodynamics of Ginzburg-Landau models. Lett. Math. Phys. 22(1):63-80, (1991). 